Supplementary Material for

\title{
Archetypical Modeling and Amphiphilic Behavior of Cobalt(II)- containing Soft-Materials with Asymmetric Tridentate Ligands
}

Rajendra Shakya, Sarmad Sahiel Hindo, Libo Wu, Marco Allard, Mary Jane Heeg, Hrant P. Hratchian, Bruce R. McGarvey, Sandro R. P. da Rocha, Claudio N. Verani *

\section{Cartesian Coordinates and Energies of the Optimized Structures}

\begin{tabular}{|c|c|c|c|}
\hline \multicolumn{4}{|l|}{$\begin{array}{l}\left(1^{\mathrm{H}}-C i s\right) \\
E=-2758.142376 \text { a.u. }\end{array}$} \\
\hline $\mathrm{Co}$ & -0.00007400 & -0.00044700 & 0.15483400 \\
\hline $\mathrm{N}$ & -1.50083900 & 1.54953200 & 0.17547400 \\
\hline $\mathrm{C}$ & -1.59392000 & 2.41029300 & -0.85112500 \\
\hline $\mathrm{H}$ & -0.73739400 & 2.41361700 & -1.51826900 \\
\hline $\mathrm{C}$ & -2.70347500 & 3.23041400 & -1.03505100 \\
\hline $\mathrm{H}$ & -2.74124900 & 3.91225400 & -1.87845700 \\
\hline $\mathrm{C}$ & -3.75729300 & 3.14296900 & -0.12530100 \\
\hline $\mathrm{H}$ & -4.64513200 & 3.75791500 & -0.24445300 \\
\hline $\mathrm{C}$ & -3.66096500 & 2.24653100 & 0.93640000 \\
\hline $\mathrm{H}$ & -4.46675000 & 2.14178700 & 1.65696900 \\
\hline $\mathrm{C}$ & -2.51078800 & 1.46605200 & 1.05527700 \\
\hline $\mathrm{C}$ & -2.28435100 & 0.53232900 & 2.22743600 \\
\hline $\mathrm{H}$ & -1.76468100 & 1.09525900 & 3.01569900 \\
\hline $\mathrm{H}$ & -3.24950600 & 0.21479400 & 2.64694300 \\
\hline $\mathrm{N}$ & -1.43591900 & -0.61601600 & 1.86012500 \\
\hline $\mathrm{H}$ & -0.89573700 & -0.88596800 & 2.68052300 \\
\hline $\mathrm{C}$ & -2.21783900 & -1.82357200 & 1.44758600 \\
\hline $\mathrm{H}$ & -2.85470900 & -2.15489900 & 2.28265700 \\
\hline $\mathrm{H}$ & -1.46853500 & -2.60279400 & 1.26036300 \\
\hline C & -3.05754700 & -1.59964500 & 0.22408400 \\
\hline $\mathrm{C}$ & -4.44744700 & -1.75143600 & 0.25408300 \\
\hline $\mathrm{H}$ & -4.93070900 & -2.00012900 & 1.19914000 \\
\hline C & -5.21730500 & -1.60740800 & -0.89948800 \\
\hline $\mathrm{C}$ & -4.57064600 & -1.30883000 & -2.10572800 \\
\hline $\mathrm{H}$ & -5.15514500 & -1.19796700 & -3.01724700 \\
\hline $\mathrm{C}$ & -3.19020200 & -1.15189300 & -2.15839000 \\
\hline $\mathrm{C}$ & -2.39053200 & -1.28583200 & -0.99660400 \\
\hline 0 & -1.08449800 & -1.15589100 & -1.03849700 \\
\hline $\mathrm{N}$ & 1.50102400 & -1.55015300 & 0.17590800 \\
\hline $\mathrm{C}$ & 1.59441100 & -2.41114800 & -0.85046000 \\
\hline $\mathrm{H}$ & 0.73772700 & -2.41521400 & -1.51739600 \\
\hline $\mathrm{C}$ & 2.70447400 & -3.23057300 & -1.03443200 \\
\hline $\mathrm{H}$ & 2.74248400 & -3.91264300 & -1.87764100 \\
\hline $\mathrm{C}$ & 3.75848000 & -3.14213600 & -0.12499200 \\
\hline $\mathrm{H}$ & 4.64670700 & -3.75650900 & -0.24420800 \\
\hline $\mathrm{C}$ & 3.66183100 & -2.24544900 & 0.93646900 \\
\hline $\mathrm{H}$ & 4.46774400 & -2.13993000 & 1.65678000 \\
\hline
\end{tabular}


$\mathrm{C}$
$\mathrm{C}$
$\mathrm{H}$
$\mathrm{H}$
$\mathrm{N}$
$\mathrm{H}$
$\mathrm{C}$
$\mathrm{H}$
$\mathrm{H}$
$\mathrm{C}$
$\mathrm{C}$
$\mathrm{H}$
$\mathrm{C}$
$\mathrm{C}$
$\mathrm{H}$
$\mathrm{C}$
$\mathrm{C}$
$\mathrm{O}$
$\mathrm{H}$
$\mathrm{H}$
$\mathrm{H}$

(1 ${ }^{\mathrm{H}}$-Trans)

$E=-2758.145994$ a.u.
2.51114900

2. 28431700

3. 24932100

1. 76496500

1.43524000

0.89493600

2. 21652600

2.85295100

1.46679000

3.05675400

4. 44654200

4. 92928600

5.21692000

4.57090000

5.15580200

3.19059500

2. 39039200

1. 08449800

6.29548600

2. 68770600

$-6.29597300$

$-2.68682200$
-1.46572500
-0.53184800
-0.21368200
-1.09484800
0.61596900
0.88579800
1.82385700
2.15591000
2.60254300
1.59995600
1.75274600
2.00219600
1.60876100
1.30921100
1.19837400
1.15127200
1.28512700
1.15413900
1.73379300
0.92353600
-1.73165800
-0.92492700

1. 05541800

2.22737200

2. 64673100

3.01579200

1.85981200

2. 68017000

1. 44700800

2. 28212600

1.25928100

0.22385300

0.25426900

1.19939100

$-0.89895300$

$-2.10530100$

$-3.01656500$

$-2.15838200$

$-0.99693800$

$-1.03930900$

$-0.86219000$

$-3.09456800$

$-0.86306000$

$-3.09450000$
H
C
H
H
H
C
H
C
H
C
H
H
H
O
C
C
O
C
C
H
C
C
H
C
C
C
C
O
C
O
CO
H
C

$$
\begin{array}{r}
3.07619200 \\
3.07619200 \\
5.22586300 \\
0.92596100 \\
-2.60516200 \\
4.26438800 \\
-0.85719700 \\
1.87501200 \\
-1.29184600 \\
-1.79246700 \\
-3.92850600 \\
2.63700900 \\
-3.71674200 \\
-1.60321700 \\
-3.20617600 \\
4.19886100 \\
1.80422900 \\
2.48329800 \\
-2.05947400 \\
-2.66002600 \\
1.33768300 \\
-2.79893300 \\
5.10238900 \\
2.94861100 \\
-3.43646200 \\
3.43646200 \\
-1.08928000 \\
-0.00040600 \\
1.08928000 \\
0.00040600 \\
0.00000000 \\
-5.10238900 \\
-2.94861100
\end{array}
$$

$-0.32685200$

$-0.32685200$

$-0.32685200$

$-0.29700100$

$-1.91162500$

$-0.32792400$

$-2.09304900$

$-0.31210400$

0.14125700

$-1.77122700$

$-3.35924700$

5.01682200

0.03390500

$-0.32230400$

$-3.40970600$

$-0.31853800$

$-0.31592600$

4.34180000

$-2.61178300$

1.44600600

3.55556200

0.39181000

$-0.30390700$

$-0.31579600$

$-4.27456700$

4.27456700

$-2.65803900$

$-1.91970600$

2.65803900

1.91970600

0.00000000

0.30390700

0.31579600
$-4.37224300$

$-3.28712200$

$-3.06316300$

$-3.11525100$

$-2.44709400$

$-2.55740800$

$-2.19452600$

$-2.58535900$

$-2.28364400$

$-1.71702600$

$-1.24185400$

$-2.50483100$

$-1.44774500$

$-1.43243700$

$-0.42704100$

$-1.16718700$

$-1.24872200$

$-1.66479000$

$-0.49624000$

$-1.22374800$

$-1.62152900$

$-0.95813900$

$-0.56506000$

$-0.54517900$

0.64223800

$-0.64223800$

0.55299200

0.53217700

$-0.55299200$

$-0.53217700$

0.00000000

0.56506000

0.54517900 


$\begin{array}{lrrr}\text { C } & -2.48329800 & -4.34180000 & 1.66479000 \\ \mathrm{C} & -1.33768300 & -3.55556200 & 1.62152900 \\ \mathrm{C} & 2.79893300 & -0.39181000 & 0.95813900 \\ \mathrm{C} & 3.20617600 & 3.40970600 & 0.42704100 \\ \mathrm{H} & 2.66002600 & -1.44600600 & 1.22374800 \\ \mathrm{C} & -4.19886100 & 0.31853800 & 1.16718700 \\ \mathrm{C} & 2.05947400 & 2.61178300 & 0.49624000 \\ \mathrm{H} & -2.63700900 & -5.01682200 & 2.50483100 \\ \mathrm{~N} & -1.80422900 & 0.31592600 & 1.24872200 \\ \mathrm{H} & 3.71674200 & -0.03390500 & 1.44774500 \\ \mathrm{H} & 3.92850600 & 3.35924700 & 1.24185400 \\ \mathrm{~N} & 1.60321700 & 0.32230400 & 1.43243700 \\ \mathrm{C} & 1.79246700 & 1.77122700 & 1.71702600 \\ \mathrm{H} & 1.29184600 & -0.14125700 & 2.28364400 \\ \mathrm{C} & -4.26438800 & 0.32792400 & 2.55740800 \\ \mathrm{C} & -1.87501200 & 0.31210400 & 2.58535900 \\ \mathrm{H} & -5.22586300 & 0.32685200 & 3.06316300 \\ \mathrm{H} & 0.85719700 & 2.09304900 & 2.19452600 \\ \mathrm{H} & 2.60516200 & 1.91162500 & 2.44709400 \\ \mathrm{H} & -0.92596100 & 0.29700100 & 3.11525100 \\ \mathrm{C} & -3.07619200 & 0.32685200 & 3.28712200 \\ \mathrm{H} & -3.07619200 & 0.32685200 & 4.37224300 \\ \mathrm{H} & 4.33280700 & 4.88752300 & -0.67429200 \\ \mathrm{H} & 0.59337200 & 3.61107000 & -2.41246900 \\ \mathrm{H} & -4.33280700 & -4.88752300 & 0.67429200 \\ \mathrm{H} & -0.59337200 & -3.61107000 & 2.41246900 \\ & & & \\ & & & \end{array}$
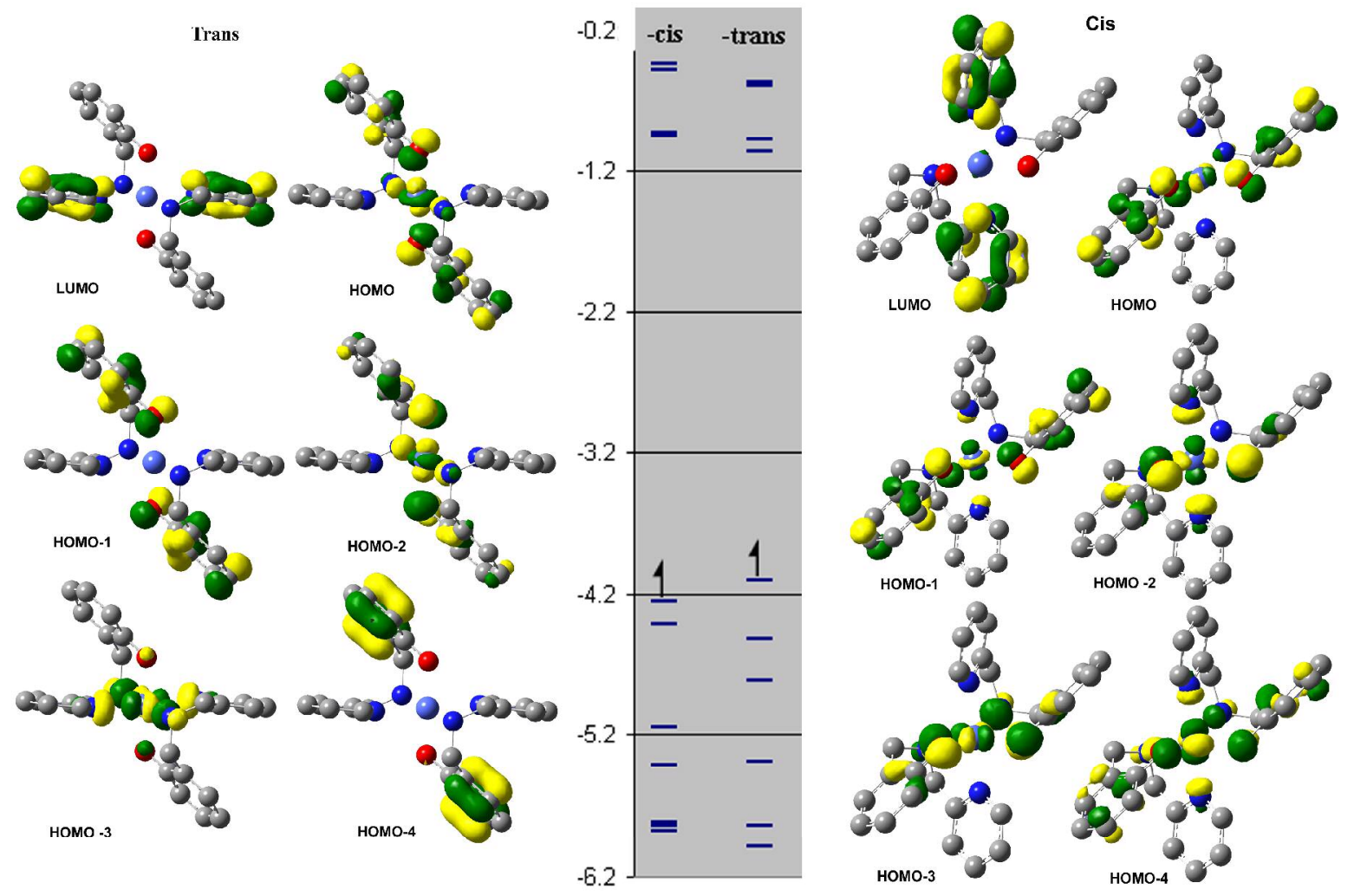